\title{
Care Giving As a Chronic Psychosocial Distress: Neuroinflammation Trajectory
}

\section{Hassanin HI}

Geriatric Medicine \& Gerontology department, Faculty of Medicine, Ain Shams University, Cairo, Egypt.

\section{Introduction and epidemiology:}

All causes dementia are progressively increasing across the globe and according to statistics in 2015, the number of people with dementia was predicted to be 47 million worldwide [1]. As we know, Alzheimer's disease (AD) constitutes 55-60\% of dementia etiology and it is a gradually progressive neurodegenerative disease, not only affect the cognitive function of the patient but also affect the functional status and interferes his/her independence. So, by time and disease progression there is a strong positive association between disease severity and the necessity for caregiving. Recent researches showed that $60-70 \%$ of caregivers are women and most of them are informal or unpaid caregivers with escalating need for structured support system either to themselves and/or their patients.

Additionally, the financial and economic burden of informal caregiving was highly exceeding the cost of professional caregiving in daycare and nursing home facilities. In a study done by Hurd and his colleagues in 2013, it was found that the average cost of informal caregiving reaches $\$ 56290$ annually per patient [2] and the total number is expected to be massively increased because the approximate number of family caregivers is expected to reach 37 million by 2050 with more than $85 \%$ increase from 2000 [3].

The role of an informal caregiver (i.e. a person who provide care to a person with a disability, not in a professional context) is a probable source of considerable psychosocial stress. Patients with cognitive impairment may depend progressively upon his/her informal caregivers, most commonly would be a family members, those caregivers either choose their current role with free well or found to be obligated to do, and usually help their relatives with dementia in their activities of daily living, as well as potential facing a challenging behavior e.g. BPSD (Behavioral And Psychological Symptoms of Dementia) and safety issues. In addition to escalating levels of stress, family dementia caregivers express increased levels of anxiety and a wide range of depressive symptoms [4].

Regarding a specific definition for caregiver burden, there is no approved one regarding the International Classification of Diseases, Ninth Revision (ICD-9) or ICD-10 code [5] but, in a longitudinal study of husbands and wives as caregivers, Zarit and colleagues offered a useful definition: "The extent to which caregivers perceive that caregiving has had an adverse effect on their emotional, social, financial, physical, and spiritual functioning."[6]. The previous definition highlights the multidimensional perception of caregiving on care providers and also that caregiving is a unique experience perceived differently from one to another[7]. Many caregiving situations probably cause burden and distress for caregivers (e.g., the need for heavy assistance with activities of daily living, social isolation, and financial deprivation as a result of illness and caregiving), but different thresholds exist for triggering caregiver burden. On the other hand, Caregiving in certain situations could be emotionally rewarding because it might tighten family bonds, honor past service the caregiver received from the person now needing care, and save family resources [8].

Caregiver burden is a term used to describe a weight or load which could be distinguished to the subjective and objective burden. Objective burden refers to the physical assistance provided by caregivers in the form of caregiving tasks that are performed. Subjective burden refers to the psychological, social, and emotional impact on caregivers as a consequence of objective burden [9]

\section{Predictors of caregiving distress:}

Caregiver's stress is better to be described as a multidimensional difficulty [10]. Well-being usually formulated from a balance and dynamic interaction between variable stressors and available resources. This mixture is unique from one caregiver to the next and dynamic over time. Therefore, multidimensional approaches are more effective than one-dimensional 
programs because the latter is more likely to tackle one particular caregiver's problem [11].

BPSD are accounted to be one of the major sources of distress for the caregivers [12], [13] and considered to be a strong prediction of breakdown of care at home [14] but it is the 'BPSD-related distress', rather than the presence of BPSD themselves [15] because frequent BPSD are not essentially the most distressing factor for caregivers [16].

Additionally, in the context of understanding caregiver burden, Campbell et al. (2008) described that neither cognitive function, level of dependence nor the presence of BPSD are significant predictors of caregiver burden. Rather, caregiver psychosocial factors, such as a sense of competence, quality of relationship with their relative and personality (neuroticism), are the principal predictors of burden [17].

Furthermore, personality characteristics of the caregiver were found to impact BPSD-related distress through burden as a mediator [18] and that is constant with the study concluded that several psychosocial characteristics related to the caregivers themselves were principally influenced the scale of burden perception to caregiver distress. Caregiver reports of relationship quality, guilt feeling, perceived sense of competence, reactivity to BPSD and burden predicted the association between BPSD frequency and BPSDrelated distress[19].

In addition to that, some researchers address other vulnerable caregivers to distress like advanced age of caregivers [20], women [21] and same residency with the patient experience greater burden than male caregivers, younger age and those who live apart from the care-recipient numerous studies have reported that spousal caregivers experience the highest level of burden [22], which could be explained by former causes, social isolation and withdrawal from social activity are considered to be major predictors of caregiver burden and outcomes of caregiver burden [23]. Lastly, lack of choice in becoming a caregiver associated with caregiver burden [24].

There is a controversy regarding the gender difference as a risk factor for caregiver distress, in an early systematic review done in 2008, which studied 93 articles discussing the gender difference, most of them conclude that female gender is tended to experience more psychological distress and utilization of more resources but in terms of worry and anxiety there were a contradicting findings [25]. Furthermore, several studies have reported no gender differences [26]. There is lack of knowledge on gender differences in continuous caregiving affecting distress and wellbeing of dementia caregivers but in many cultures, the women are considered as caregivers and men as breadwinners. It is considered as their duty to care for the family.

In a very interesting study regarding patients with Parkinson Disease as a neurodegenerative disease like
$\mathrm{AD}$ and their caregivers it was found that Caregiverburden showed steady escalating levels with longer duration, advanced severity and disability of the disease, in particular manifestation of cognitive impairment, depression, confusion and hallucinations, as well as falls which are associated with the greatest impact on caregiver quality of life, and more attention should be paid to caregivers' emotional and physical health, particularly in advancing PD with psychiatric complications and falls. Their surprising finding also demonstrates that patients' and caregivers' depression and quality of life scores are closely related, highlighting the importance of viewing patient and caregiver as one unit. Management of caregiver distress as mentioned before should be multidimensional as the psychosocial aspects of caregivers, should be included in the management of PD in order to improve patient and caregiver lives and to decrease the probability of nursing home placement [27].

\section{Outcomes of caregiver distress:}

Unfortunately, caregiver burden and psychosocial distress have an extensive range of clinical pictures and consequences like, anxiety, Suicidal thoughts, financial stress [28], increased risk of mortality as chronic exposure to caregiver burden identified as an independent predictor of caregiver mortality with a $63 \%$ increased risk of death [29] and this is suggested to be due to broad range of metabolic and psychological detrimental implications. Also, family caregivers who experience higher levels of stress could have a reduction in their life expectancy by 10 years [30] as well as increased risk of work absence and social life withdrawal.

For instance, caregiver distress could be associated with either intentional or non-intentional weight loss, with or without loss of appetite as a feature of classical depression and/or anxiety, financial distress and/or chronic fatigue; sleep deprivation is one of the most popular drawbacks of caregiving of dementia patients who have disruption of the sleep-wake cycle or suffering from BPSD.

Additionally, depression which has a bidirectional relationship with caregiver burden with a resultant poor self-care and of ignoring self-health [31], inability to continue regular employment, Caregiving may preclude regular employment; when more than 1 potential caregiver exists, the family member who is not regularly employed is more likely to assume the caregiving role.

Nevertheless, as regard, the biological impact of caregiving burden, a recent systematic review, identified 151 papers, including papers examining differences between informal caregivers of people with dementia and controls as well as interferences aimed at the moderation of the biological burden of chronic 
caregiving stress. Results showed that stress-related hormones like cortisol levels were increased in caregivers in a majority of studies considering the measurement of this biomarker. On the other hand, there was mixed evidence for differences in epinephrine, norepinephrine (NE) and other markers of cardiovascular risk factors. There was a high level of heterogeneity in immune system measures. Caregivers performed more poorly on attention and executive functioning tests. While memory performance was variable. Examined interventions to lessen stress improved cognition but showed miscellaneous outcome on cortisol. Given the rising need for family caregivers worldwide, the implications of these findings can no longer be neglected [32].

Lastly, There is accumulating evidence that having a healthy and psychologically stable caregiver is associated with better outcome in terms of morbidity, mortality and quality of life of the patient [33], on the other hand, caregiver distress is a major predictor for institutionalization [34].

\section{Moderators of caregiver distress:}

There are a lot of conflicting results about effective measures which could moderate the caregiver burden.

For instance, cognitive behavioral therapy interventions (CBTs) as one of the most popular interventions to alleviate caregiver burden, in a systematic review and meta-analysis done in 2016 it was found that, CBTs for family or Informal Caregivers had a negligible effect and number of suggestions could be hypothesized to explain this negligible effect, and it is suggested that future researches and studies should move beyond concurrent standard CBT methods and point towards recent advances in the basic affective sciences in order to better appreciate and treat the psychological distress experienced by informal caregivers [35].

Additionally, in the clinical trial, data were analyzed from 308 family caregivers: 153 caregivers underwent 5 weeks of structured psychosocial intervention and a 3-month conversation group on the caregiver's burden and satisfaction in dementia care, which was followed up over 6 and 12 months and 155 control caregivers who did not. Repeated measures were carried out 6 and 12 months later.

Caregivers who underwent the psychosocial intervention (5-week program and 3-month conversation group) reported significantly lower tension and frustration after 6 months, and this trend remained after 12 months. These findings emphasize the importance of identifying family caregivers early in the caring process to optimize well-being. This study demonstrates that psychosocial intervention with a clearly defined aim that includes giving information and having a conversation group have significant, positive effects on burden and satisfaction for caregivers of people with dementia[36].

Regarding the role of spirituality to moderate caregiver burden, it was found that, many family members which became informal caregivers usually turn to spirituality for support and that religiosity may be an essential part of how caregivers could accept the reality of their own relative become affected with serious cognitive impairment. In particular, our data indicate that religiosity is negatively associated with symptoms of depression and is also related to how caregiving family members feel about and care for themselves. Collaborative partnerships between mental health professionals and religious and spiritual communities represent a powerful and culturally sensitive resource for meeting the needs of family caregivers of persons with serious mental illness[37]

\section{Stress-induced chronic inflammation:}

During the last few decades, numerous studies have been explored the impact of chronic psychological stress on enhancing the probability of chronic low grade of inflammatory response using experimental protocols to examine this hypothesis in mediators of inflammation after response to short-term laboratory stressors designed to simulate transient stresses of daily life. Early findings suggest that acute stress induces reliable changes in both enumerative and functional aspects of the immune system, including increases in both circulating and stimulated markers of inflammation [38]. Subsequently, those individuals who are exposed to increased levels of inflammatory cytokines have a higher probability of chronic systemic inflammation, with possibly greater risk for inflammatory disease [39].

A possible mechanism to explain these phenomenon, in a recent animal model clinical trial it was found that chronic exposure to mild stress activates microglia via monoamine neurotransmitters e.g., NE released by sympathetic neurons in the locus coeruleus [40] which provides the primary source of NE to the forebrain, mediates memory and attention [41] and it is highly sensitive to stress and emotional challenge[42]. This chronic sympathetic overstimulation by the repeated psychological challenge in mice leads to induction of microglial proinflammatory genes, such as IL-1 $\beta$ and TNF- $\alpha$ at an early stage while activates IL- 6 at a late stage, and CD14 and Cx3cr1 throughout the stress procedure [43].

In contrast, blocking of sympathetic activity by propranolol and guanethidine counteracted [43], whereas $\beta$-adrenergic stimulation by isoproterenol amplified [44], the stress-induced upregulation of proinflammatory cytokines, such as IL-1 $\beta$, in the brain. Also, chronic stress affects synaptic functions of microglia and promotes microglial secretion of IL-1 $\beta$, IL-18 and TNF- $\alpha$ [45], [46], which dose-dependently affected neuronal plasticity[47]. 
Additionally, in another animal model experiment done in 2014, it is found that, chronic exposure to different types of stress exaggerates the microglial activation and subsequent death of dopaminergic neurons after inflammatory challenge to ventral midbrain suggesting the critical role of stress for progression of neurodegenerative disorders like Parkinson disease [48] and so, applying measures that aim to reduce stress may have a role to slow the disease progression.

In humans, inflammatory response to psychosocial stress was escalated in patients with major depression exposed to early-life stress[49]. Moreover, perivascular macrophages and the proportion of primed versus resting microglia in the white matter of dorsal anterior cingulate cortex (dACC) - apart from the frontal cortex regulating decision-making, emotion and mood [50]were increased in severe depression with suicidal ideation [51]. A recent meta-analysis on post-mortem human brains demonstrated associations of suicidal behavior with inflammatory cytokines, kynurenine pathway and microgliosis in the orbitofrontal cortex, a brain region involved in suicidal vulnerability[52].Such findings identify the kynurenine pathway as a potential therapeutic target for both neurodegenerative and stress-related neuropsychiatric disorders [53]. This was further highlighted by a recent evidence that both blocking the skeletal kynurenine pathway and reducing plasma kynurenine protected mice from stress-induced depression, thereby opening therapeutic avenues for the treatment of depression by targeting this pathway without the need to cross the blood-brain barrier (BBB) [54].

A large number of research studies that examined the impact of continuous exposure to caregiver stress, job stress and burnout, low socioeconomic status, catastrophic life events, lack of social support, loneliness, and so forth and subsequent implication on the circulating serum levels of inflammatory cytokines. Many of those research studies have demonstrated that chronic stress increases the pro-inflammatory cytokine [57],[59].

There are fewer studies about chronic stress impacting on the cytokines compared to studies about that on chronic hormones, especially that the cytokines involved are relatively limited, including IL-1, IL-2, IL-6, TNF- $\alpha$, INF- $\gamma$, EGF, VEGF, TGF- $\alpha$, and so forth, while other cytokines are less involved. This might be because there are few cytokines at high circulating levels in vivo, compared to other stress hormones, especially in asymptomatic individuals [57].

For instance, in a meta-analysis of more than 300 studies about chronic stress, and they have found an increased production of IL-6 and INF- $\gamma$ during the chronic stress, compared with the control groups [58]. Hence, it is obvious that there is a conflict between the mechanism of chronic stress acting on inflammatory cytokines and the outcomes observed in quite a few studies. These inconsistencies are suggested to be results of the type, duration and intensity of stressors, detection methods, and individual differences [59].

\section{Different cytokines responses to chronic stress:}

In a recent meta-analysis done in 2017 [60], examined 34 studies that measured circulating inflammatory markers and 15 studies that measured stimulated the production of inflammatory markers before and after exposure to laboratory stressful challenge. Results showed significant stress-related increases in circulating interleukin IL-1 $\beta$, IL-6, IL-10, and TNF- $\alpha$ with high statistical significance $(\mathrm{p}<.001)$, but not IL$1 \mathrm{ra}$, IL-2, interferon- $\gamma$, or C-reactive protein. For stimulated inflammatory markers, results showed stress-related increases in IL-1 $\beta$ when measured 20-120 min post-stress $(p<.001)$, and in IL-4 and interferon- $\gamma$ when measured $0-10$ min post stressor $(p<.001)$. These results extend findings from a prior meta-analysis [38] show reliable increases in circulating IL-6, IL-1 $\beta$, IL10 and TNF- $\alpha$ and stimulated IL-1 $\beta$, IL-4 and interferon- $\gamma$ in response to acute stress. It is possible that these responses contribute to associations between exposure to life challenges and vulnerability to inflammatory disease.

However, caregivers who experience high levels of stress have demonstrated lower immune reactivity in response to challenges than non-caregivers controls [61]. In particular, in caregivers of persons with dementia, altered levels of IL-1, elevations of IL-6, and differences in rates of IL- 6 increase over time have been found [55], [62]. Caregivers of persons with cancer, however, likely have heightened stress responses (or activation of physiologic stress pathways), condensed into a shorter time span.

Controversial evidence regarding serum levels of inflammatory cytokines in response to stress: Chronic Stress may also lead to down-regulation of Pro-inflammatory Cytokines in some circumstances:

Chronic psychosocial burden is shown to be associated with continued activation of stress hormones like Glucocorticoids (GCs) and catecholamines (CAs) release that act on their classic cytoplasmic/nuclear receptors on Antigen Presenting Cells (APCs) to suppress the production of IL- 12 that considered the main inducer of Th1 responses. So, Th1 to Th2 shift will be promoted; therefore, the proinflammatory cytokines secretion will be suppressed, which are served as the main reason that the activated HPA axis and SNS axis inhibit the pro-inflammatory state [63].

In vitro studies, a negative dose-dependent relationship between GCs and pro-inflammatory cytokines was found, such as IL-6 and IL-8 [64]. Additionally, in a study done to examine the effect of 
caregiving on parents of children suffering from cancer, it was found that GCs inhibit production of IL- $1 \beta$, IL6 , and TNF- $\alpha$ as well and this finding was present in both case and control group [65]. As regard the signal transduction level, GCs and CAs also inhibit the inflammation related pathways, including NF- $\kappa \mathrm{B}$, AP1, JAK-STAT and MAPKs [66]. In conclusion, there is a repeated observation that chronic stress up-regulates different hormones, such as CRH, ACTH, GCs, and CAs [67], supporting that chronic stress down regulates the pro-inflammatory cytokines.

Additionally, an animal model study about the effect of chronic exposure to stress, it was found that, cytokines imbalance with clear shift of T-helper1 (Th1) cytokines such as INF- $\gamma$ to T-helper2 (Th2) cytokines like IL-4 and IL-10 due constant elevation of GCs and alteration of HPA axis with another interesting finding, that this Th1 to Th2 shift is associated with increased probability of cancer progression like cancer colon for instance [68].

Th1 cells primarily secrete IL- 2 , IL-6, TNF- $\alpha$, and INF- $\gamma$, which activate cytotoxic $\mathrm{T}$ cells, natural killer cells, and macrophage and further promote the cellular immunity, whereas Th2 cells secrete a different set of cytokines, primarily IL-4, IL-10, and IL-13, which promote humoral immunity. Therefore, Th1 to Th2 shift is associated with decreased immune system surveillance mechanism and balance [69], [70].

\section{The biological and metabolic impact of chronic inflammation:}

There is seminal evidence that chronic elevation of inflammatory cytokines has major consequences and impact on both biological and psychological status of the patients.

For instance, overexpression of proinflammatory cytokines has been associated with several neuropsychiatric disorders, such as anxiety, sleep disturbance[71], depression [72][73] and neurodegenerative diseases, including $\mathrm{AD}$ and Parkinson's disease [74].

Patients with major depressive disorder (MDD) exhibit both increased immune activation and dysregulated activity of neuronal plasticity, neurogenesis [26] and abnormal cellular immunity [75].In addition to a recent meta-analysis have reported significant upregulation of IL-1b, IL-6, and TNF-a, in both serum and plasma of patients with depression [76].As regard IL-1 $\beta$ which is one of the key cytokines in neuroinflammation that show increased levels after chronic exposure to stress, produces a reduction in neurogenesis in the adult hippocampus, and this effect is associated with the development of anhedonia(one of the key features of depressive symptoms)[77]. In contrast, administration of an IL-1 $\beta$ receptor antagonist abolishes both the antineurogenic and the anhedonic behavioural effects of chronic unpredictable stress [78]. Moreover, dysregulation of adult hippocampal neurogenesis is crucial to the development of depressive behavior and the ability to cope with stress [79] and that is constant with the emerging evidence suggests that, during an inflammatory response, cytokines influence the neurogenic niche and regulate neural progenitor cells (NPCs) proliferation and neurogenesis, particularly in the context of psychiatric and neurodegenerative conditions [80].

Furthermore, Neurogenesis is a complex neurobiological process by which new neurons are generated from neural stem cells (NSCs)[81]. Approximately 700 new neurons are added to the adult human hippocampus daily, suggesting that adult hippocampal neurogenesis has a critical role in mediating human brain functions, such as memory formation and cognition [82], [83].

Unfortunately, this essential dynamic neurogenesis process has very restricted capabilities in the adult life to fix and repair any damage in CNS tissue [84]. NSCs and NPCs undergo a dynamic state of stimulation to divide, maturate, differentiate and proliferate. On the hand, any disturbance in the internal environment of the brain or any neurodegenerative pathological, infection or injurious condition, for instance, will escalate and activate a cascade of molecular and cellular pathways that lead to microglial activation and priming with the subsequent uprising of inflammatory state and elevated levels of cytokines [85]. Cytokines can have a substantial role in the brain: they can confer immune protection, clearing the system from dead and damaged neurons, on the other hand, they can exert a certain detrimental effect on the neural plasticity[75].By contrast, activation of peripheral inflammation is associated with increased expression of cytokines in the neurogenic niches [86], which directly impairs hippocampal-dependent forms of synaptic plasticity [87], potentially leading to cognitive impairment [88].

Moreover, those proinflammatory cytokines distribute to the peripheral circulation and stimulate the hepatocytes to secrete acute-phase reactive reactants (such as CRP, serum amyloid A, Haptoglobin), and trigger the HPA axis, interrelate with the neural transmitters, and induce behavioral changes: the " sickness behavior " (such as easy fatigability, depressive mood, and cognitive decline). All the above reactions subserve the metabolic demand of inflammation [89]. At the cellular levels, NF- $\square \mathrm{B}$ is activated by kinds of pathways to boost the proinflammatory mediators secretion, including proinflammatory cytokines (IL-1 $\square$, IL-6, TNF- $\square$, IFN$\square)$, chemokines, Cellusr Adesion Molecules, and Acute Phase Proteins [89].

Persistent elevation of inflammatory cytokines is associated with many biological changes on the neurotransmitter physiological pathways inside the CNS, dopamine and serotonin for instance, might undergo suppression and reuptake blockade and 
escalating the probability of having depressive symptoms [90] in addition to disruption of synaptic plasticity through altered release of (BDNF) [91]. Finally, the concerns about the relationship between inflammation and ageing have been raised in the current years. IL-6, TNF- $\square$, and IL-1 $\square$, and other proinflammatory cytokines are overexpressed in variety of age-related diseases and are positively associated with age.

From chronic psychosocial distress to neuroinflammation trajectory:

Accumulating evidence and research have been suggested the induction of series of changes and dysregulation that affects the immune system after chronic exposure to stress with subsequently altered mood and personal behaviour [92].

For instance, chronic exposure to stress like psychosocial burden may activate a "transcriptional fingerprint" on the peripheral leucocytes and hence upregulation of pro-inflammatory transcriptional control pathways like NF-k $\beta$ [93][94] and that mechanism participates into elevated peripheral levels of inflammatory cytokines like IL-6, IL- $1 \beta$ and TNF- $\alpha$ in the blood of subjects with affective disorders [95].

On the other hand, one of the most common neurodegenerative disorders like $\mathrm{AD}$ with the chief pathological finding of abnormal $\mathrm{A} \beta$ accumulation and tau tangles showing up the microglial sensitivity to inflammatory stimuli [96]. These supposed phenomena of "microglial priming" characterized by the production of cytokines IL-1, IL-18 and TNF- $\alpha$ and release of nitric oxide [97] and that is may exacerbate $A \beta$ accumulation and neuronal loss, resulting in a cycle of microglial priming and release of pro-inflammatory cytokines, with a subsequent escalation of neurodegeneration [97].In a study conducted on more than 74,000 AD participants, showing overrepresentation of immune-inflammatory genes contributes to $\mathrm{AD}[98]$.

This 'vicious cycle' of neuroinflammation and neurodegeneration may enhance microglial proinflammatory activation and impair their ability to clear A $\beta$. Over the past two decades, many therapeutic trials on various $\mathrm{AD}$ immunotherapies have been developed to remove $A \beta$ plaques, inhibit $A \beta$ aggregation and deposition, or reduce the production of $A \beta$ in the brain via inhibition of $\alpha$-secretase and $\gamma$-secretase, trialled both pre-clinically and clinically. Although antibodies directed against $A \beta$ can successfully clear plaques and reverse cognitive deficits in mouse models, all these strategies, however, failed in clinical trials [99],[100].

The failure of these immunotherapy trials suggests that targeting $\mathrm{A} \beta$ alone may not be enough to prevent or slowdown AD progression, as multiple mechanisms are involved in AD pathogenesis, and their relative contributions may vary at different stages of the disease, among which neuroinflammation is a key component [101].

Inflammaging and neuroinflammation :

Inflammation in CNS or what is called Neuroinflammation is a widely spreading phenomenon in all kinds of neurodegenerative conditions, such as Amyotrophic Lateral Sclerosis, Parkinson's disease, Alzheimer's disease and Primary Progressive Multiple Sclerosis, which has a progressive detrimental impact on neurocognitive function. The etiology of many of those diseases is primarily sporadic, without specific cellular or soluble components can contribute to inflammatory response [102].

Despite the different trajectory pattern of each neurodegenerative disease is behaving, neuroinflammation is almost always a prominent feature in the disease progression. At the moment, in certain cases, it was believed that localized neuroinflammatory response considered as a reflection of the ongoing chronic systemic inflammatory condition.

In addition to, the immunological privileged pattern of CNS and on bases of the destructive behavior of the neuroinflammation on the brain parenchyma, led to the common view that entry of circulating immune cells to the CNS could only escalate the parenchymal damage, and therefore, many trials have been tested to use systemic anti-inflammatory drugs to mitigate neuroinflammation in neurodegenerative diseases [103].

Microglia, are resident macrophage located in the CNS which playing a crucial role in the immune defence of this system [104] that phagocytosing toxic elements in the CNS[105], previously named by the Spanish neuroscientist, Pío del Río Hortega about a century ago [105]. Activated microglia, like peripheral macrophages, are often classified into inflammatory (M1) and alternatively activated (M2) phenotypes [106]. However, these cells show high levels of diversity and plasticity and their classification into an M1 or M2 polarized state may be an oversimplification [107]. Recently, it has been proposed that microglia switch in a continuous fashion between phenotypes [108].

M1 phenotype is activated and stimulated by means of interferon (IFN) $\gamma$ and LPS stimulation while, M2 phenotype by means of interleukin IL-4, IL-10, and IL13 [109]. M1 microglia are associated with the production and release of pro-inflammatory cytokines, namely TNF- $\alpha$, IL-6, IL-23, IL-1 $\beta$, IL-12, nitric oxide In addition to chemokines[110]. In contrast, M2 microglia possess anti-inflammatory profile and potentiate production of $\mathrm{IL}-10$ and TGF- $\beta$, and extracellular matrix molecules [111].In addition, it has been proposed that M1 microglia predominate at the site of injury under pathological situations, whereas M2 microglia appear later at a stage more related to repair 
phase [112].

It is important to mention that, commonly known neurodegenerative disorders, $\mathrm{AD}$, for instance, exhibit mixed profile of activation of both phenotypes M1 and M2 [113]. Nevertheless, elderly people show dysfunction and dystrophia in the immune cells in CNS [112], which includes local changes of cytokine expression [114].

Furthermore, chronic inflammation and the delirium accompanying severe systemic infection have been shown to be risk factors for AD in the elderly, and vice versa, several risk factors for $\mathrm{AD}$ are also inducers of systemic inflammation [115]. As a consequence, levels of cytokines, their receptors and other proteins associated with immune responses in blood and CSF of AD patients have been frequently investigated to uncover mechanisms of neuroinflammation in dementia or in the context of biomarker research.

\section{Neuroinflammation might increase the probability of cognitive impairment:}

For many years since in 1991, pathogenic mutations in the amyloid protein precursor (APP) in rare cases of familial AD were discovered [116], then the "amyloid cascade hypothesis" was proposed and came to the light [117]. According to this hypothesis, production of toxic $\mathrm{A} \beta$ aggregates is the major pathway in the pathogenesis of AD. The discovery of AD-related mutations in presenilin 1 and 2[118], which are catalytic subunits of the $\gamma$-secretase complex involved in APP cleavage and $\mathrm{A} \beta$ production [119], further supported the hypothesis.

The amyloid-inflammatory cascade hypothesis also comes in front after repeated research suggestions of the presence of inflammatory cytokines both locally in the brain and peripherally in the serum blood. For instance, in the metanalysis done by Walter and his colleagues in 2010 about comparing the cytokine concentrations in both CSF and peripheral blood of Alzheimer patients [120] it was found that, in peripheral blood, there were significantly higher concentrations of IL- $6,(p<.00001)$, TNF- $\alpha(p<.01)$, $\mathrm{N}$, IL-1 $\beta,(\mathrm{p}<.00001)$, TGF- $\beta,(\mathrm{p}<.0006)$, IL-12, $(\mathrm{p}<$ $.00001)$, and IL-18, $(\mathrm{p}<.03)$, but on the other hand not of IL-4, IL-8, IL-10, interferon- $\gamma$, or C-reactive protein in $\mathrm{AD}$ subjects compared with control subjects. There were significantly higher concentrations of TGF- $\beta$, ( $p$ $<.006$ ), but not IL-6, TNF- $\alpha$, and IL-1 $\beta$ in the CSF of AD subjects compared with control subjects. Beside other studies high pointed the elevated levels of cytokines in the CSF as risk factors for deterioration of mild cognitive impairment to the dementia stage of $\mathrm{AD}$ or as a sign of disease progression and more deterioration of cognitive function[121].

Furthermore, the neurodegenerative trajectory of $\mathrm{AD}$ in the brain tissue shows cytokines, complement defence, acute phase reactants, a manifestation of microglia priming and activation as well as elevated levels of TNF- $\alpha$ in cerebrospinal fluid [122].

Also, in another study that aimed to track different trajectories of inflammatory markers and cognitive impairment over 10 years especially (IL-6) and (CRP) trajectory components (slope, variability, and baseline level) and among 1,323 adults, age 70 to 79 years. It was found that, extreme CRP variability was significantly associated with cognitive decline (HR 1.6, 95\% CI: 1.1-2.3) and the association remained among women ( $\mathrm{HR}=1.8 ; 95 \% \mathrm{CI}: 1.1,3.0)$ and among those with no APOE e4 allele $(\mathrm{HR}=1.6 ; 95 \% \mathrm{CI}$ : 1.1, 2.5). There were no significant associations between slope or baseline level of CRP and cognitive decline, nor between IL-6 and cognitive decline [124]. One possible explanation of that, CRP variability may reflect a greater burden of vascular and metabolic disease, and perhaps signify conditions that are more poorly controlled [124].

Moreover, in terms of using anti-inflammatory therapeutics as adjunctive options in AD, many studies especially in animal models like the study done by Jun Yin in 2017 examined the relationship between neuroinflammation and disease progression in $\mathrm{AD}$, in that study the researchers used JC-124, which a small molecule that inhibits NOD-Like Receptor Protein 3 (NLRP3) inflammasome, inhibits the caspase-1 cleavage and activation in the TgCRND8 mice. NLRP3 is a core molecule in the pathway of neuroinflammation which leads to the final elaboration of IL- $1 \beta$ and IL-18 that considered potent activators of inflammatory profile in the brain. The results analysis conclude that inhibition of (NLRP3) and caspase-1 cleavage effectively reduced amyloid deposition and alleviated AD-associated deficits [123].

Those finding and after exploration of the pivotal role of microglia in $\mathrm{A} \beta$ phagocytosis and clearance, we have a strong potential evidence of the concordance role of neuroinflammation in potentiation and even worsening of neurodegenerative course of AD. In particular, the failure of a clinical study of $A \beta$ vaccination, immunotherapies with the antibodies, and other studies with $\mathrm{A} \beta$ targeted drugs strongly suggest that the amyloid hypothesis at least does not provide a sufficient basis for the development of therapeutic drugs[125]. Even some clinical trials of using $\mathrm{A} \beta$ vaccination showed that elimination of $A \beta$ had no effect on the spread of tau pathologies or the progression of AD.

\section{References}

1. [M. Prince, M., Wimo, A., Guerchet, M., Ali, G., Wu, Y.T., Prina "The Global Impact of Dementia. An Analysis of Prevalence Incidence, Cost and Trends," London, UK, 2015.

2. L. K. Hurd MD, Martorell P, Delavande A, Mullen KJ, "Monetary costs of dementia in the United States," N Engl J Med., vol. 368, no. 14, pp. 1326-1334, 2013

3. L. Ho et al., "Biomarkers of Resilience in Stress Reduction for 
Caregivers of Alzheimer???s Patients," NeuroMolecular Med., vol. 18, no. 2, pp. 177-189, 2016.

4. [G. Mahoney, R., Regan, C., Katona, C., Livingston, "Anxiety and depression infamily caregivers of people with Alzheimer disease: the LASER-AD study," Am. J.Geriatr. Psychiatry, vol. 13, pp. 795801,2005

5. "ICD - ICD-10-CM - International Classification of Diseases, Tenth Revision, Clinical Modification." [Online]. Available https://www.cdc.gov/nchs/icd/icd10cm.htm. [Accessed: 13-Sep2017].

6. Z. J. Zarit SH, Todd PA, "Subjective burden of husbands and wives as caregivers: a longitudinal study," Gerontologist., vol. 26, no. 3 , pp. 260-266, 1986.

7. GillickMR., "The critical role of caregivers in achieving patientcentered care," JAMA2, vol. 310, no. 6, pp. 575-576, 2013

8. M. S. L. RonaldD. Adelman, Lyubov L. Tmanova, DVM, MLIS Diana Delgado, Sarah Dion, "Caregiver Burden," JAMA, vol. 311, no. 10, pp. 1052-1059, 2014.

9. [B. E. Montgomery RV, Stull DE, ".Measurement and the analysis of burden," Res Aging, vol. 7, pp. 137-152, 1985.

10. Aneshensel, C. S., "Profiles in caregiving: the unexpected career," San Diego, CA Acad. Press., 1995

11. S. Zarit and E. Femia, "Behavioral and Psychosocial Interventions for Family Caregivers," J. Soc. Work Educ., vol. 44, no. sup3, pp. 49-57, 2008

12. P. A. Craig D, Mirakhur A, Hart DJ, Mcllroy SP, "A cross-sectional study of neuropsychiatric symptoms in 435 patients with Alzheimer's disease," Am. J. Geriatr. psychiatry, vol. 13, pp. 460$468,2005$.

13. [13] et al. Bandeira DR, Pawlowski J, Goncalves TR, "Psychological distress in Brazilian caregivers of relatives with dementia," Aging Ment Heal., vol. 11, pp. 14-19, 2007.

14. [14] et al Gaugler JE, Wall MM, Kane RL, "Does caregive burden mediate the effects of behavioral disturbances on nursing home admission?," Am J Geriatr Psychiatry, vol. 19, pp. 497-506, 2011.

15. R. P. Chan DC, Kasper JD, Black BS, "Presence of behavioral and psychological symptoms predicts nursing home placement in community-dwelling elders with cognitive impairment in univariate but not multivariate analysis," J Gerontol A Biol Sci Med Sci, vol. 58, pp. 548-554, 2003.

16. G. A. Fauth EB, "Which behavioral and psychological symptoms of dementia are the most problematic? Variability by prevalence, intensity, distress ratings, and associations with carer depressive symptoms," Int J Geriatr Psychiatry, vol. 29, pp. 263-271, 2014

17. Campbell $P$, Wright J, Oyebode J, "Determinants of burden in those who care for someone with dementia," Int J Geriatr Psychiatry, vol. 23, pp. 1078-1085, 2008.

18. de M. A. Melo G, Maroco J, "Influence of personality on caregiver's burden, depression and distress related to the BPSD," Int J Geriat Psychiatry, vol. 26, pp. 1275-1282, 2011.

19. A. Feast, M. Orrell, I. Russell, G. Charlesworth, and E. Moniz Cook, "The contribution of caregiver psychosocial factors to distress associated with behavioural and psychological symptoms in dementia," Int. J. Geriatr. Psychiatry, pp. 76-85, 2016.

20. L.-B. J. \& Y.-L. V. Serrano-Aguilar P.G., "Impact on health-related quality of life and perceived burden of informal caregivers of individuals with Alzheimer's disease," Neuroepidemiology, vol. 27, no. 3, pp. 136-142, 2006

21. J. L. \& L. C. Campbell P., Wright J., Oyebode J., Job D., Crome P., Bentham P., "Determinants of burden in those who care for someone with dementia. International Journal of Geriatric," Psychiatry, vol. 23, no. 10, pp. 1078-1085, 2008.

22. V.-F. J. \& L.-P. S. Conde-Sala J.L., Garre-Olmo J., Turro-Garriga O., "Differential features of burden between spouse and adult-child caregivers of patients with Alzheimer's disease: an exploratory comparative design," Int. J. Nurs. Stud., vol. 47, no. 10, pp. 12621273, 2010.

23. S. R. Rodakowski J, Skidmore ER, Rogers JC, "Role of social support in predicting caregiver burden," Arch Phys Med Rehabil, vol. 93, no. 12, pp. 2229-2236, 2012

24. M. J. Schulz R, Beach SR, Cook TB, Martire LM, Tomlinson JM, "Predictors and consequences of perceived lack of choice in becoming an informal caregiver.," Aging Ment Heal., vol. 16, no. 6, pp. 712-721, 2012.

25. N. Baker, K. L \& Robertson, "Coping with caring for someone with dementia: reviewing the literature about men," Aging Ment. Heal., vol. 12, pp. 413-422, 2008.

26. D. Baker, K. L., Robertson, N., \& Connelly, "Men caring for wives or partners with dementia: masculinity, strain and gain," Aging
Ment. Heal., vol. 14, pp. 319-327, 2010

27. A Schrag, A. Hovris, D. Morley, N. Quinn, and M. Jahanshahi, "Caregiver-burden in parkinson's disease is closely associated with psychiatric symptoms, falls, and disability," Park. Relat. Disord. vol. 12, no. 1, pp. 35-41, 2006.

28. E. K. Salmon JR, Kwak J, Acquaviva KD, Brandt K "Transformative aspects of caregiving at life's end," $J$ Pain Symptom Manag., vol. 29, no. 2, pp. 121-129, 2005.

29. B. S. Schulz R, "Caregiving as a risk factor for mortality: the Caregiver Health Effects Study," JAMA, vol. 282, no. 23, pp. 22152219, 1999.

30. P. S. Arno, "Economic value of informal caregiving. Paper presented at the Care Coordination and the Caregiving Forum of the Department of Veterans Affairs Employee Education System and the Office of Care Coordination, Bethesda, MD," Economic value of informal caregiving. Paper presented at the Care Coordination and the Caregiving Forum of the Department of Veterans Affairs Employee Education System and the Office of Care Coordination, Bethesda, MD, 2006. [Online]. Available: www.thefamilycaregiver.org/ who/stats.cfm. [Accessed: 11-Jan2007].

31. M.-L. C. Hoffman GJ, Lee J, "Health behaviors among baby boomer informal caregivers," Gerontologist, vol. 52, no. 2, pp. 219230, 2012.

32. A. P. Allen et al., A systematic review of the psychobiological burden of informal caregiving for patients with dementia: Focus on cognitive and biological markers of chronic stress, vol. 73. Elsevier Ltd, 2017.

33. [33] T. B. Dickens CM, McGowan L, Percival C, Douglas J and C. F. Cotter L, Heagerty A, "Lack of a close confidant, but not depression, predicts further cardiac events after myocardial infarction," Heart, vol. 90, pp. 518-522, 2004.

34. G. CJ, "Influence of emotional distress among supporters on the outcome of psychogeriatric day care.," Br J Psychiatry, vol. 150, pp. 219-223, 1987.

35. M. S. O'Toole, R. Zachariae, M. E. Renna, D. S. Mennin, and A. Applebaum, "Cognitive behavioral therapies for informal caregivers of patients with cancer and cancer survivors: A systematic review and meta-analysis," Psychooncology., 2016.

36. A. Signe, S. Elmståhl, S. Andren, S. Elmstahl, and A. Signe "Psychosocial intervention for family caregivers of people with dementia reduces caregiver's burden: Development and effect after 6 and 12 months," Scand. J. Caring Sci., vol. 22, no. 1, pp. 98$109,2008$.

37. A. B. Murray-Swank, A. Lucksted, D. R. Medoff, Y. Yang, K Wohlheiter, and L. B. Dixon, "Religiosity, psychosocial adjustment, and subjective burden of persons who care for those with mental illness.," Psychiatr. Serv., vol. 57, no. 3, pp. 361-365, 2006.

38. Y. Steptoe, A., Hamer, M., Chida, "The effects of acute psychological stress on circulating inflammatory factors in humans: A review and meta-analysis.," Brain Behav. Immun2, vol. 21, no. 7 , pp. 901-912, 2007.

39. P. J. Lockwood, K.G., Marsland, A.L., Cohen, S., Gianaros, "Sex differences in the association between stressor-evoked interleukin6 reactivity and C-reactive protein," Brain Behav. Immun, vol. 58, pp. 173-180, 2016.

40. S. Delpech, J.C., Madore, C., Nadjar, A., Joffre, C., Wohleb, E.S. Laye, "Microgliain neuronal plasticity: influence of stress," Neuropharmacology, vol. 96, pp. 19-28, 2015.

41. S. J. Sara, "The locus coeruleus and noradrenergic modulation of cognition," Nat. Rev. Neurosci., vol. 10, no. 3, pp. 211-223, Mar. 2009.

42. S. C. Kelly, B. He, S. E. Perez, S. D. Ginsberg, E. J. Mufson, and S. E. Counts, "Locus coeruleus cellular and molecular pathology during the progression of Alzheimer's disease," Acta Neuropathol. Commun., vol. 5, no. 1, p. 8, Dec. 2017

43. J. F. McKim, D.B., Patterson, J.M., Wohleb, E.S., Jarrett, B.L., Reader, B.F., Godbout, J.P., Sheridan, "Sympathetic Release of Splenic Monocytes PromotesRecurring Anxiety Following Repeated Social Defeat.," Biol. Psychiatry, vol. 79, pp. 803-813, 2016.

44. [L. T. Johnson, J.D., Zimomra, Z.R., Stewart, "Beta-adrenergic receptor activationprimes microglia cytokine production," J. Neuroimmunol., vol. 254, pp. 161-164, 2013

45. R. Goshen, I., Kreisel, T., Ben-Menachem-Zidon, O., Licht, T., Weidenfeld, J., Ben-Hur, T., Yirmiya, "Brain interleukin-1 mediates chronic stress-induceddepression in mice via adrenocortical activation and hippocampalneurogenesis suppression.," Mol. Psychiatry, vol. 13, pp. 717-728, 2008.

46. R. Rossetti, A.C., Papp, M., Gruca, P., Paladini, M.S., Racagni, G. Riva, M.A., Molteni, "Stress-induced anhedonia is associated with 
the activation of theinflammatory system in the rat brain: Restorative effect of pharmacologicalintervention.," Pharmacol. Res., vol. 103, pp. 1-12, 2016

47. G. Bobula, B., Sowa, J., Hess, "Anti-interleukin-1beta antibody prevents theoccurrence of repeated restraint stress-induced alterations in synaptictransmission and long-term potentiation in the rat frontal cortex.," Pharmacol.Rep., vol. PR 67, pp. 123-128, 2015.

48. R. M. de Pablos et al., "Chronic stress enhances microglia activation and exacerbates death of nigral dopaminergic neurons under conditions of inflammation," J. Neuroinflammation, vol. 11, no. 1 , p. 34, 2014

49. C. M. Pace, T.W., Mletzko, T.C., Alagbe, O., Musselman, D.L. Nemeroff, C.B., Miller, A.H., Heim, "Increased stress-induced inflammatory responses in malepatients with major depression and increased early life stress," Am. J. Psychiatry, vol. 163, pp. 1630 1633, 2006.

50. G. Bush et al., "Dorsal anterior cingulate cortex: a role in rewardbased decision making.," Proc. Natl. Acad. Sci. U. S. A., vol. 99 , no. 1, pp. 523-8, Jan. 2002

51. N. Torres-Platas, S.G., Cruceanu, C., Chen, G.G., Turecki, G., Mechawar, "Evidence for increased microglial priming and macrophage recruitment in the dorsal anterior cingulate white matter of depressed suicides.," Brain Behav.Immun., vol. 42, pp. 50-59, 2014

52. D. Courtet, P., Giner, L., Seneque, M., Guillaume, S., Olie, E. Ducasse, "Neuroinflammation in suicide: toward a comprehensive model," World J. Biol.Psychiatry, vol. 17, pp. 564-586, 2016.

53. A. O'Farrell, K., Harkin, "Stress-related regulation of the kynurenine pathway:Relevance to neuropsychiatric and degenerative disorders.," Neuropharmacology2, vol. 112, pp. 307323, 2017

54. R. Agudelo, L.Z., Femenia, T., Orhan, F., Porsmyr-Palmertz, M., Goiny, M., Martinez-Redondo, V., Correia, J.C., Izadi, M., Bhat, M., Schuppe-Koistinen, I., Pettersson, A.T., Ferreira, D.M., Krook, A., Barres, R., Zierath, J.R., Erhardt, S., Lindskog, M., "Skeletal muscle PGC-1alpha1 modulateskynurenine metabolism and mediates resilience to stress-induced depression," Cell, vol. 159, pp. 33-45, 2014.

55. Kiecolt-Glaser JK et al, "Chronic stress and age-related increases in the proinflammatory cytokine IL-6," Proc Natl Acad Sci USA, vol. 100, no. 15, pp. 9090-9095, 2003.

56. J. K.-G. J. P. Gouin, R. Glaser, W. B. Malarkey, D. Beversdorf "Chronic stress, daily stressors, and circulating inflammatory markers," Heal. Psychol., vol. 31, no. 2, pp. 264-268, 2012

57. R. von K. A. Hansel, S. Hong, R. J. A. Camara, "Inflammation as a psychophysiological biomarker in chronic psychosocial stress," Neurosci. Biobehav. Rev., vol. 35, no. 1, pp. 115-121, 2010.

58. G. E. M. S. C. Segerstrom, "Psychological stress and the human immune system: a meta-analytic study of 30 years of inquiry," Psychol. Bull., vol. 130, no. 4, pp. 601-630, 2004.

59. R. M. S. S. F. Sorrells, J. R. Caso, C.D.Munhoz, "The stressed CNS: when glucocorticoids aggravate inflammation," Neuron, vol. 64, no. 1, pp. 33-39, 2009

60. N. A. Marsland, A.L., Walsh, C., Lockwood, K., John-Henderson, "The effects of acute psychological stress on circulating inflammatory factors in humans: A review and meta-analysis," Brain. Behav. Immun., 2017.

61. Kiecolt-Glaser JK et al, "Chronic stress alters the immune response to influenza virus vaccine in older adults," Proc Natl Acad Sci USA, vol. 93, no. 7, pp. 3043-3047, 1996.

62. Lutgendorf SK et al, "Life stress, mood disturbance, and elevated interleukin-6 in healthy older women.," J Gerontol Ser A Biol SCi Med Sci, vol. 54, no. 9, pp. 434-439, 1999.

63. C. L. R. and A. H. Miller, "When not enough is too much: the role of insufficient glucocorticoid signaling in the pathophysiology of stress-related disorders," Am. J. Psychiatry, vol. 160, no. 9, pp. $1554-1565,2003$

64. K. M. J. J. Oppenheim, C. O. C. Zachariae, N. Mukaida "Properties of the novel proinflammatory supergene 'intercrine' cytokine family," Annu. Rev. Immunol., vol. 9, pp. 617-648, 1991.

65. A. K. R. G. E. Miller, S. Cohen, "Chronic psychological stress and the regulation of pro-inflammatory cytokines: a glucocorticoidresistance mode," Heal. Psychol., vol. 21, no. 6, pp. 531-541, 2002.

66. J. A. C. J. M. Busillo, K. M. Azzams, "Glucocorticoids sensitize the innate immune systemthrough regulation of theNLRP3 inflammasome,"” J. Biol. Chem., vol. 286, no. 44, pp. 3870338713, 2011

67. R. G. and J. K. Kiecolt-Glaser, "stress-induced immune dysfunction: implications for health," Nat. Rev. Immunol., vol. 5, no. 3, pp. 243-251, 2005.

68. N. Hou et al., "A novel chronic stress-induced shift in the Th1 to Th2 response promotes colon cancer growth," Biochem. Biophys. Res. Commun., vol. 439, no. 4, pp. 471-476, 2013.

69. S. C. S. and G. E. Miller, "Psychological stress and the human immune system: a meta-analytic study of 30 years of inquiry," Psychol. Bull., vol. 130, no. 4, pp. 601-630, 2004.

70. I. J. E. and G. P. Chrousos, "Stress hormones, proinflammatory and antiinflammatory cytokines, and autoimmunity," Ann. N. Y. Acad. Sci., vol. 966, pp. 290-303, 2002.

71. D. Kovacs, P. Kovacs, N. Eszlari, X. Gonda, and G. Juhasz "Psychological side effects of immune therapies: symptoms and pathomechanism," Curr. Opin. Pharmacol., vol. 29, pp. 97-103 2016.

72. P. A. Zunszain, et al. "Inflammation and Depression," Curr. Top. Behav. Neurosci., vol. 14, pp. 135-151, 2013.

73. Hany I. Hassanin, "Late life Depression: Prevalence, Pathogenesis, Clinical Presentation, Evaluation and Emerging Therapeutics, Egypt. J. Geriatr. Gerontol., vol. 2, no. 2, pp. 1-11, 2015.

74. N. L. Monson et al., "Elevated CNS Inflammation in Patients with Preclinical Alzheimer's Disease," J. Cereb. Blood Flow Metab., vol. 34, no. 1, pp. 30-33, Jan. 2014

75. S. Das and A. Basu, "Inflammation: A new candidate in modulating adult neurogenesis," J. Neurosci. Res., vol. 86, no. 6, pp. 1199 1208, May 2008

76. Y. Dowlati et al., "A Meta-Analysis of Cytokines in Major Depression," Biol. Psychiatry, vol. 67, no. 5, pp. 446-457, 2010.

77. D. R. Koo JW, "IL-1beta is an essential mediator of the antineurogenic and anhedonic effects of stress. U S A," Proc Natl Acad Sci, vol. 105, pp. 751-756, 2008

78. D. R. Koo JW, Russo SJ, Ferguson D, Nestler EJ, "Nuclear factorkappaB is a critical mediator of stress-impaired neurogenesis and depressive behavior," Proc Natl Acad Sci U S A, vol. 107, no. 6, pp. 2669-2674, 2010 .

79. C. Anacker and C. M. Pariante, "Can adult neurogenesis buffer stress responses and depressive behaviour?," Mol. Psychiatry, vol. 17, no. 1, pp. 9-10, Jan. 2012.

80. A. Fuster-Matanzo et al., "Role of neuroinflammation in adult neurogenesis and Alzheimer disease: therapeutic approaches.," Mediators Inflamm., vol. 2013, p. 260925, 2013.

81. I. Imayoshi, M. Sakamoto, T. Ohtsuka, and R. Kageyama "Continuous neurogenesis in the adult brain," Dev. Growth Differ. vol. 51, no. 3, pp. 379-386, Feb. 2009.

82. K. L. Spalding et al., "Dynamics of Hippocampal Neurogenesis in Adult Humans," Cell, vol. 153, no. 6, pp. 1219-1227, 2013.

83. W. Deng, J. B. Aimone, and F. H. Gage, "New neurons and new memories: how does adult hippocampal neurogenesis affect learning and memory?," Nat. Rev. Neurosci., vol. 11, no. 5, pp. 339-350, May 2010

84. T. Honda, $H$. Namiki, M. Kudoh, $H$. Nagase, and H. Mizutani, "A Formal Total Synthesis of Securinine via an Intramolecular [4+2] Cycloaddition Reaction," Heterocycles, vol. 59, no. 1, p. 169, 2003.

85. Y. Shigemoto-Mogami, K. Hoshikawa, J. E. Goldman, Y. Sekino, and K. Sato, "Microglia Enhance Neurogenesis and Oligodendrogenesis in the Early Postnatal Subventricular Zone," J. Neurosci., vol. 34, no. 6, 2014.

86. R. Dantzer, J. C. O'Connor, G. G. Freund, R. W. Johnson, K. W. Kelley, and K. K. Dantzer R, O'Connor JC, Freund GG, Johnson RW, "From inflammation to sickness and depression: when the immune system subjugates the brain," Nat Rev Neurosci, vol. 9 no. 1, pp. 46-56, Jan. 2008.

87. J. McAfoose and B. T. Baune, "Evidence for a cytokine model of cognitive function," Neurosci. Biobehav. Rev., vol. 33, no. 3, pp. 355-366, 2009.

88. C. J. Wilson, C. E. Finch, and H. J. Cohen, "Cytokines and Cognition-The Case for A Head-to-Toe Inflammatory Paradigm," J Am. Geriatr. Soc., vol. 50, no. 12, pp. 2041-2056, Dec. 2002.

89. A. H. M. C. L. Raison, L. Capuron, "Cytokines sing the blues: inflammation and the pathogenesis of depression," Trends Immunol., vol. 27, no. 1, pp. 24-31, 2006.

90. T. A. A. J. Dunn, J. Wang, "Effects of cytokines on cerebral neurotransmission: comparison with the effects of stress," Adv. Exp. Biol., vol. 461, no. 117-127, 1999.

91. S. K. et al. T. Lu, Y. Pan, "Gene regulation and DNA damage in the ageing human brain," Nature, vol. 429, no. 6994, pp. 883-891, 2004

92. R. C. Miller AH, Maletic V, "Inflammation and its discontents: the role of cytokines in the pathophysiology of major depression," Biol Psychiatry, vol. 65, no. 9, pp. 732-741, 2009. 
93. S. S. Cole SW, Conti G, Arevalo JM, Ruggiero A, Heckman JJ, "Transcriptional modulation of the developing immune system by early life social adversity," Proc Natl Acad Sci U S A, vol. 109, pp. 20578-20583, 2012.

94. R. B. Powell ND, Sloan EK, Bailey MT, Arevalo JM, Miller GE, Chen E, Kobor MS and C. S. Sheridan JF, "Social stress upregulates inflammatory gene expression in the leukocyte transcriptome via $\beta$-adrenergic induction of myelopoiesis.," Proc Natl Acad Sci U S A, vol. 110, no. 41, pp. 16574-16579, 2013.

95. A. O. Dahl J, Ormstad H, Aass HC, Sandvik L, Malt UF, "Recovery from major depressive disorder episode after non-pharmacological treatment is associated with normalized cytokine levels," 2016.

96. C. Perry, V.H., Holmes, "Microglial priming in neurodegenerative disease.," Nat.Rev. Neurol., vol. 10, pp. 217-224, 2014.

97. M. T. Heneka et al., "NLRP3 is activated in Alzheimer's disease and contributes to pathology in APP/PS1 mice," Nature, vol. 493, pp. 674-678, 2013

98. P. Lambert, J.C., Ibrahim-Verbaas, C.A., Harold, D., Naj, A.C. Sims, R., Bellenguez, C., DeStafano, A.L., Bis, J.C., Beecham, G.W., Grenier-Boley, B., Russo, G., Thorton-Wells, T.A., Jones, N., Smith, A.V., Chouraki, V., Thomas, C., Ikram, M.A., Zelenika, D, "Meta-analysis of 74,046 individuals identifies 11 newsusceptibility loci for Alzheimer's disease.," Nat Genet, vol. 45, pp. 1452-1458, 2013.

99. I. Liu, E., Schmidt, M.E., Margolin, R., Sperling, R., Koeppe, R., Mason, N.S., Klunk, W.E., Mathis, C.A., Salloway, S., Fox, N.C. Hill, D.L., Les, A.S., Collins, P., Gregg, K.M., Di, J., Lu, Y., Tudor, I.C., Wyman, B.T., Booth, K., Broome, S., Yuen, E., Gr, "Amyloidbeta11C-PiB-PET imaging results from 2 randomized bapineuzumab phase 3 AD trials.," Neurology, vol. 85, pp. 692 $700,2015$.

100. R. Siemers, E.R., Sundell, K.L., Carlson, C., Case, M., Sethuraman, G., Liu-Seifert, H., Dowsett, S.A., Pontecorvo, M.J., Dean, R.A., Demattos, "Phase 3solanezumab trials: secondary outcomes in mild Alzheimer's disease patients," Alzheimers Dement, vol. 12, pp. 110-120, 2016.

101. I. Krstic, D., Knuesel, "Deciphering the mechanism underlying lateonsetAlzheimer disease," Nat. Rev. Neurol., vol. 9, pp. 25--34, 2013.

102. T. M. Frank-Cannon TC, Alto LT, McAlpine FE, "Does neuroinflammation fan the flame in neurodegenerative diseases?," Mol NeurobiolNeurodegener, vol. 4, no. 47, 2009.

103. S. R. Schwartz M, "Systemic inflammatory cells fight of neurodegenerative disease," Nat Rev Neurol, vol. 6, pp. 405-410, 2010.

104. G. S. Martinez FO, "The $M 1$ and $M 2$ paradigm of macrophage activation: time for reassessment," Prime Rep, vol. 6, p. 13, 2014

105. S. A. Tremblay ME, Lecours C, Samson L, Sanchez-Zafra V, "From the Cajal alumni Achucarro and Rio-Hortega to the rediscovery of never-resting microglia," Front Neuroanat, vol. 9, p. 45, 2015

106. N. J. Boche D, Perry VH, "Activation patterns of microglia and their identification in the human brain," Neuropathol Appl Neurobiol, vol. 39, pp. 3-18, 2013

107. R. Ransohoff, "A polarizing question: do $M 1$ and $M 2$ microglia exist?," Nat Neurosci, vol. 19, pp. 987-991, 2016.

108. M. T. Heneka et al., "Neuroinflammation in Alzheimer's disease," Lancet Neurol., vol. 14, no. 4, pp. 388-405, 2015.

109. H. G. Orihuela R, McPherson CA, "Microglial M1/M2 polarization and metabolic states," $\mathrm{Br} J$ Pharmacol, vol. 173, pp. 649-665 2016.

110. B. K. Loane DJ, "Role of microglia in neurotrauma," Neuro Ther., vol. 7, pp. 366-377, 2010

111. D. Michell-Robinson MA, Touil H, Healy LM, Owen DR and M. C. BA, Bar-Or A, Antel JP, "Roles of microglia in brain development, tissue maintenance and repair," Brain, vol. 138, pp. 1138-1159, 2015.

112. L. W. Tang $Y$, "Differential Roles of $M 1$ and $M 2$ Microglia in Neurodegenerative Diseases," Mol Neurobiol, vol. 53, pp. 1181 1194, 2016.

113. V. M. Colton CA, Mott RT, Sharpe H, Xu Q, Van Nostrand WE "Expression profiles for macrophage alternative activation genes in $A D$ and in mouse models of $A D, " J$ Neuroinflamm, vol. 3, no. 2, p. $27,2006$.

114. Tarkowski $\mathrm{E}$ et al .." "Intrathecal inflammation precedes development of Alzheimer's disease.," J Neurol Neurosurg Psychiatry, vol. 74, no. 9, pp. 1200-1205, 2003.

115. K. J. Bettcher BM, "Inflammation and clinical presentation in neurodegenerative disease: a volatile relationship," Neurocase, vol. 19, no. 2, pp. 182-200, 2012.
116. L. Goate, A.; Chartier-Harlin, M.C.; Mullan, M.; Brown, J.; Crawford, F.; Fidani, L.; Giuffra, L.; Haynes, A.; Irving, N.; James, et al "Segregation of a missense mutation in the amyloid precursor protein gene with familial Alzheimer's disease," Nat. 1, vol. 349, pp. 704-706, 1991.

117. S. D. J. Hardy J., "The amyloid hypothesis of Alzheimer's disease: Progress and problems on the road to therapeutics," Science (80-. )., vol. 297, pp. 353-356, 2002.

118. T. Rogaev, E.I.; Sherrington, R.; Rogaeva, E.A.; Levesque, G Ikeda, M.; Liang, Y.; Chi, H.; Lin, C.; Holman, K.; Tsuda. et al., "Familial Alzheimer's disease in kindreds with missense mutations in a gene on chromosome 1 related to the Alzheimer's disease type 3 gene," Nature, vol. 376, pp. 775-778, 1995.

119. D.Wolfe, M.S.; Xia, W.; Ostaszewski, B.L.; Diehl, T.S.; Kimberly, W.T.; Selkoe, "Two transmembrane aspartates in presenilin-1 required for presenilin endoproteolysis and -secretase activity," Nature, vol. 398, pp. 513-517, 1999.

120. W. Swardfager, K. Lanctt, L. Rothenburg, A. Wong, J. Cappell, and N. Herrmann, "A meta-analysis of cytokines in Alzheimer's disease," Biol. Psychiatry, vol. 68, no. 10, pp. 930-941, 2010.

121. S. E. Galimberti D, Fenoglio C, "Inflammation in neurodegenerative disorders: friend or foe?," Curr Aging Sci, vol. 1, pp. 30-41, 2008.

122. Mark E McCaulley and Kira A Grush, "Alzheimer's Disease: Exploring the Role of Inflammation and Implications for Treatment," Int. J. Alzheimers. Dis., vol. 2015, pp. 1-10, 2015.

123. D. J. Klionsky et al., "Guidelines for the use and interpretation of assays for monitoring autophagy (3rd edition)," Autophagy, vol. 12 , no. 1, pp. 1-222, 2016

124. A. L. Metti et al., "Trajectories of inflammatory markers and cognitive decline over 10 years," Neurobiol. Aging, vol. 35, no. 12 , pp. $2785-2790,2014$

125. X. Doody, R.S.; Thomas, R.G.; Farlow, M.; Iwatsubo, T.; Vellas, B.; Joffe, S.; Kieburtz, K.; Raman, R.; Sun and P. S. . et al. Aisen, "Phase 3 trials of solanezumab for mild-to-moderate Alzheimer's disease.," N. Engl. J. Med., vol. 370, pp. 311-321, 2014. 